Somnologie $2017 \cdot 21: 91-92$

DOI 10.1007/s11818-017-0118-4

C) Springer Medizin Verlag GmbH 2017

CrossMark

\section{Thomas Penzel ${ }^{1,2} \cdot$ Hagen Malberg}

'Lehrstuhl für Biomedizinische Technik, TU Dresden, Dresden, Deutschland

${ }^{2}$ Interdisziplinäres Schlafmedizinisches Zentrum, Charité - Universitätsmedizin Berlin, Berlin, Deutschland

\title{
Technologische Entwicklungen in der Schlafmedizin
}

In der Schlafmedizin haben sich früh technische Fortschritte der Digitalisierung und der Miniaturisierung von $\mathrm{Me}$ dizintechnik etabliert. Schlafmedizin mit der spezifischen Anforderung, den Schlaf der Patienten mit minimaler Störung und so umfassend wie möglich zu messen, hat hohe Anforderungen an medizintechnische Entwicklungen gestellt. Diese beginnen bei der Messtechnik, beinhalten Analysetechniken und gehen bis zur Technik neuer Therapien und Telemedizin. Sie bleiben aber nicht bei rein "technischen“ Entwicklungen stehen, sondern umfassen auch konzeptionelle Ansätze bezüglich eines Managements von Schlaf, wie der letzte Beitrag des Schwerpunkthefts zeigt.

In Anerkennung der Bedeutung technologischer Entwicklungen für die Schlafmedizin wurde die Jahrestagung der DGSM 2016 vom Lehrstuhl für Biomedizinische Technik der TU Dresden ausgerichtet. Prof. Dr.-Ing. Hagen Malberg gab der Tagung eine besondere Prägung und schlug die Brücke zu aktuellen technischen Innovationen in der Medizintechnik. Gemeinsam mit Prof. Malberg haben wir herausragende Beitragende von technischen Innovationen auf dem Kongress angesprochen und um die Darstellung ihrer Arbeiten für das vorliegende Schwerpunktheft gebeten.

Im ersten Beitrag führt S. Zaunseder die aktuellen Entwicklungen der berührungslosen Messung des Schlafes und seiner Möglichkeiten für die schlafmedizinische Diagnostik aus. Die klassische Schlafmessung wird vom Schlafmediziner mit der kardiorespiratorischen Polysomnographie mit einer Vielzahl von Elektroden und Kabeln gleichgesetzt. Einerseits erlaubt diese etablierte
Technik eine umfassende Erfassung des Schlafes und der physiologischen Funktionen, und andererseits kennen wir die vielen Artefakte in den aufgezeichneten Signalen und die Beeinträchtigungen des Schlafenden durch die Verkabelung. Gerade bei Patienten mit Insomniebeschwerden kann die Verkabelung zu Beeinträchtigungen führen. Daher war es immer ein Wunsch, mit weniger Elektroden am Körper oder möglicherweise sogar ohne Elektroden am Körper den Schlaf zu messen. Frühe Ansätze hierzu fanden sich bereits bei dem Einsatz des „static charge sensitive bed“ (SCSB) in den Achtzigerjahren in Finnland. Eine Übersicht über den aktuellen Stand der Technik mit ihren Möglichkeiten inklusive Mikrowellentechnik und Limitationen sowie einem weiterführenden Ausblick wird hier dargestellt.

Ebenfalls mit berührungsloser Technik befasst sich der zweite Beitrag. Herr H. Garn stellt eine dreidimensionale Videoaufnahmetechnik des schlafenden Patienten vor, welche es ermöglicht, periodische Beinbewegungen im Schlaf zu detektieren. Dies ist von großer Bedeutung für das häufige Krankheitsbild der periodischen Beinbewegungen im Schlaf (PLMS), und eine besondere Herausforderung an die Technik ist der Vergleich mit der Elektromyographie. Denn schwerlich kann eine Bildgebung eine elektrische Reizleitung abbilden. Sie kann jedoch in einer Studie verglichen und damit validiert werden. Der spannende Vergleich zeigt die Möglichkeiten und Grenzen dieser Technik an einer originalen Studie auf.

Der dritte Beitrag von C. Fischer stellt ebenfalls eine Originalarbeit dar. Unter den Folgen von Schlafstörungen nehmen die kardiovaskulären Folgeerkrankungen einen wichtigen Platz ein. Entsprechend möchte man die kardiovaskulären Folgen bereits in der Nacht erfassen und quantifizieren. Dies betrifft nicht nur die schlafbezogenen Atmungsstörungen, bei denen die Folgen für Bluthochdruck und Herzrhythmusstörungen bekannt sind, sondern auch die schlafbezogenen Bewegungsstörungen, die Insomnien und die Schlaf-Wach-Rhythmusstörungen.

Das klassische EKG-Monitoring wurde in den letzten Jahren um die Aufnahme der Pulswelle erweitert. Die Pulswelle steht relativ einfach über die pulsoximetrische Messung im Schlaflabor zur Verfügung. Die Verarbeitung der Pulswelle und das Extrahieren von Parametern, die eine kardiovaskuläre Gefährdung belegen oder sogar vorhersagen, stehen dagegen noch am Anfang. C. Fischer zeigt sehr sorgfältig und systematisch auf, wie die Pulswelle analysiert werden kann und welche Schritte der Artefakterkennung und -bearbeitung erforderlich sind, um eine valide Auswertung dieses Signals zu ermöglichen. Für die Arbeit wurde ein großes Kollektiv an Patienten herangezogen. Dieser Beitrag ist sehr solide und kann Grundlage für wichtige weiterführende Analysen der Pulswelle werden.

Der vierte Artikel von $H$. Wöhrle greift das hochaktuelle Thema der Telemedizin auf. In dieser Originalarbeit werden Daten ausgewertet und dargestellt, die telemedizinisch erhoben wurden und die Therapietreue von PAP-Behandlung bei Patienten mit schlafbezogenen Atmungsstörungen dokumentieren. Eine anonymisierte Auswertung einer sehr großen Datensammlung von behandelten Patienten erlaubt eine Auswertung und Weiterentwicklung des Therapiema- 
nagements dieser großen Patientengruppe. Die telemedizinische Therapieüberwachung erlaubt ein proaktives Management der PAP-Therapie mit frühzeitiger Rückkopplung zum Patienten. In der Folge nutzen Patienten mit diesem Management ihre Therapie im Schnitt länger und brechen ihre Therapie auch seltener ab. Sowohl aus individueller Sicht als auch aus gesundheitsökonomischer Sicht ist dies überaus begrüßenswert und sollte bedacht werden. Als offene Frage in der Studie bleibt natürlich die Kostenfrage dieser zusätzlichen telemedizinischen Überwachungsleistung.

Es ist uns eine besondere Freude, dass drei der vier Beiträge Originalarbeiten sind und somit das Gebiet der technologischen Entwicklungen in der Schlafmedizin mit großen Schritten voranbringen. Weitere Originalarbeiten wurden für dieses Thema angeworben, konnten aber nicht mehr rechtzeitig fertiggestellt werden und werden als freie Beiträge in den folgenden Heften der Somnologie erscheinen.

Es ist besonders bemerkenswert, dass drei der vier Artikel Studien präsentieren, die durch öffentliche Mittel gefördert wurden. Insofern kann hier festgestellt werden, dass öffentliche Drittmittelgeber erkannt haben, welche große Bedeutung die Medizintechnik und deren Entwicklungen in der Schlafmedizin darstellen. Dies wird von Seiten der Herausgeber außerordentlich begrüßt und erfreut festgestellt. Auf diesem Weg können Deutschland und Österreich den medizintechnischen Fortschritt weiter voran bringen.

Zusätzlich zu dem Schwerpunkt enthält das Heft weitere Beiträge. Ein Beitrag, der auch gut zum Technologieschwerpunkt passt, ist die Übersicht zur Fluglärmwirkungsforschung auf den Schlaf unter Berücksichtigung aktueller Studien.
Wir wünschen viel Freude beim Lesen dieses Somnologie-Heftes.

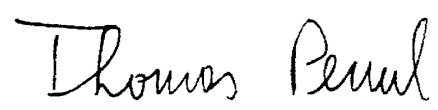

Thomas Penzel

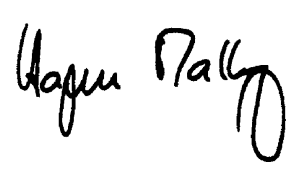

Hagen Malberg

\section{Korrespondenzadresse}

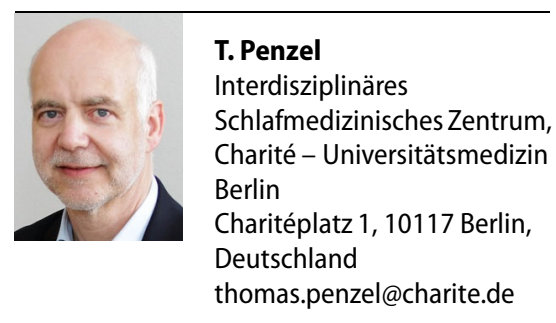

Interessenkonflikt. T. Penzel hat Unterstützung für Forschungsprojekte von Cidelec, Itamar, Resmed und Philips erhalten. Er hat Vortragshonorare von Somnodent und Inspire erhalten. H. Malberg gibt an, dass kein Interessenkonflikt besteht. 